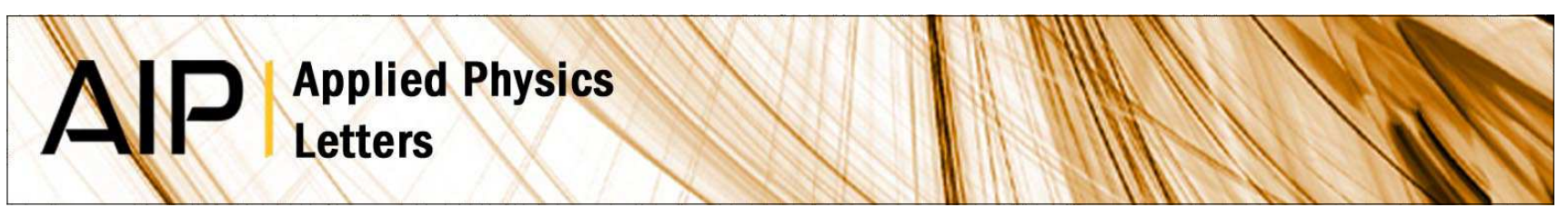

\title{
Optical properties of ultrathin InAs quantum-well-heterostructures
}

R. Samti, F. Raouafi, M. Chaouach, M. Maaref, A. Sakri et al.

Citation: Appl. Phys. Lett. 101, 012105 (2012); doi: 10.1063/1.4731783

View online: http://dx.doi.org/10.1063/1.4731783

View Table of Contents: http://apl.aip.org/resource/1/APPLAB/v101/i1

Published by the American Institute of Physics.

\section{Related Articles}

Ultraviolet emission efficiency enhancement of a-plane AIGaN/GaN multiple-quantum-wells with increasing quantum well thickness

Appl. Phys. Lett. 100, 261901 (2012)

Intense monochromatic terahertz electromagnetic waves from coherent GaAs-like longitudinal optical phonons in (11n)-oriented $\mathrm{GaAs} / \mathrm{In} 0.1 \mathrm{Al}$.9As strained multiple quantum wells

Appl. Phys. Lett. 100, 242107 (2012)

Optically addressed near and long-wave infrared multiband photodetectors

Appl. Phys. Lett. 100, 241103 (2012)

InGaN/GaN single-quantum-well microdisks

Appl. Phys. Lett. 100, 242101 (2012)

Increasing the optical transition oscillator strength in GaSb-based type II quantum wells

Appl. Phys. Lett. 100, 231908 (2012)

\section{Additional information on Appl. Phys. Lett.}

Journal Homepage: http://apl.aip.org/

Journal Information: http://apl.aip.org/about/about_the_journal

Top downloads: http://apl.aip.org/features/most_downloaded

Information for Authors: http://apl.aip.org/authors

\section{ADVERTISEMENT}

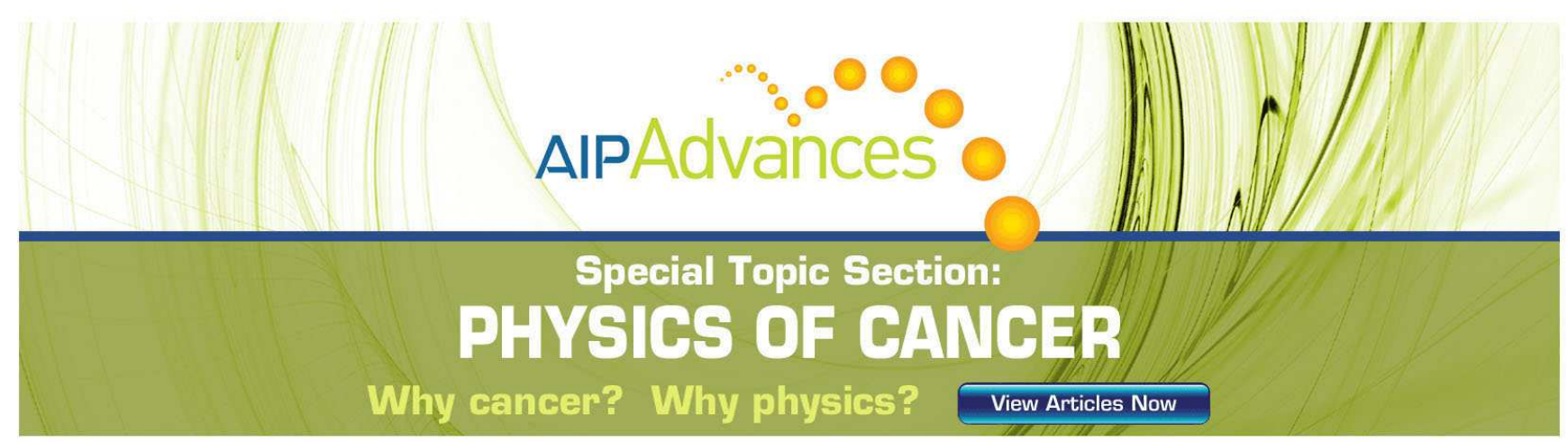




\title{
Optical properties of ultrathin InAs quantum-well-heterostructures
}

\author{
R. Samti, ${ }^{1}$ F. Raouafi, ${ }^{2}$ M. Chaouach, ${ }^{1}$ M. Maaref, ${ }^{1}$ A. Sakri, ${ }^{3}$ J. Even, ${ }^{3}$ J.-M. Gerard, ${ }^{4}$ \\ and J.-M Jancu ${ }^{3}$ \\ ${ }^{1}$ Unité de Recherche de Physique des Semi-conducteurs et Capteurs, Institut Préparatoire aux Etudes \\ Scientifiques et Techniques, BP51, 2070 La Marsa, Tunisia \\ ${ }^{2}$ Laboratoire de physico-chimie, des microstructures et des microsystèmes, Institut Préparatoire aux Etudes \\ Scientifiques et Techniques, BP51,2070 La Marsa, Tunisia \\ ${ }^{3}$ Université Européenne de Bretagne, INSA FOTON, UMR 6082, F-35708 Rennes, France \\ ${ }^{4}$ CEA INAC SP2M, Nanophys \& Semicond Lab, F-38054 Grenoble, France
}

(Received 25 April 2012; accepted 13 June 2012; published online 5 July 2012)

\begin{abstract}
Band structure calculations of complete InAs monolayer in $\mathrm{AlGaAs} / \mathrm{GaAs}$ quantum wells are performed within the framework of the extended-basis $s p^{3} d^{5} s^{*}$ tight-binding model. We show that the optical properties can be tuned from the quantum well energy below the GaAs band-gap depending on the well thickness and the position of the probe. The results are supported by differential reflectivity measurements and represent a concept for optoelectronic devices with an operation wavelength widely tuneable around $850 \mathrm{~nm}$ employing GaAs process technology. @ 2012 American Institute of Physics. [http://dx.doi.org/10.1063/1.4731783]
\end{abstract}

Semiconductor quantum wells (QWs) heterostructures have been the focus of vast research efforts in the last 30 years and the improvement of their optical properties has enabled the realization of devices through novel chemical and physical effects. Using different materials combinations it is possible to produce QW lasers over a wide wavelength range 100-3000 nm, some of them entered the commercial market less than a decade after their creation. In particular, GaAs and InAs are the building blocks of a large range of nanostructures for optical applications. However, twodimensional (defect-free) growth of InAs on $\mathrm{GaAs}(001)$ substrate is only limited to very thin layers ${ }^{1}$ because the lattice mismatch between the two materials is about of $6.8 \%$. After deposition of about 2 monolayers (MLs), the formation of quantum dots via the Stranski-Krastanow mechanism is observed. $^{2}$ It is known that strain changes both the band structure and discontinuity of the band edges in the quantum structure ensuring an additional degree of freedom for the optical and transport properties. ${ }^{3}$ For ultrathin strained InAs layers on $\operatorname{GaAs}(001)$, the heavy-hole band is pulled up and the light-hole band is pushed down giving an energy splitting as large as the energy of the split-off band. As a consequence, the in-plane hole mass of the ground state is parabolic with a light-hole character, which is desired for high-speed devices utilizing valence bands. ${ }^{3}$ A precise description of the strained valence band dispersion should include hole interactions with the split-off and lowest conduction bands and this can be obtained with the 8-band k.p model ${ }^{4}$ or atomistic approaches like the empirical pseudopotential ${ }^{5}$ and tight-binding (TB) methods. ${ }^{6}$ From the experimental side, InAs monolayers or submonolayers (SML) in bulk GaAs have demonstrated a surprisingly low-dimensional character, providing high quantum efficiencies resulting in an intense photoluminescence below the GaAs bandgap as well as enhanced spin splitting properties. ${ }^{7}$ In particular, Goñi et al. ${ }^{8}$ observed a strong laser emission from a single ML, ascribed to zero-dimensional excitonic states, in analogy to the lasing properties of semiconductor quantum dots. On the other hand, Marzin and Gerard ${ }^{9}$ succeeded in probing the probability density of the first electronic states in a GaAs/AlGaAs QW using planar layers of InAs and AlAs. The latter system was also studied by several other authors. ${ }^{10,11}$

In this paper, we demonstrate the possibility of tuning the optical properties from a single InAs monolayer inserted at different positions in a QW. The unperturbed structure consists in a GaAs well of $16 \mathrm{~nm}$ wide alternating with $\mathrm{Al}_{0.3} \mathrm{Ga}_{0.7} \mathrm{As}$ barriers grown on $\mathrm{GaAs}(001)$. In our simulations, the barriers are sufficiently thick to isolate the well. The photoluminescence and photoluminescence excitation measurements of this configuration were previously reported in Ref. 9 but the numerical modelling is still lacking. We show from atomistic simulations that the optical dipole of the lowest energy transitions strongly depends on the position of the probe in the confinement potential. We evidence parity-forbidden transitions in asymmetric structures and calculations are supported by our differential reflectivity measurements.

We consider the extended-basis $s p^{3} d^{5} s^{*}$ tight-binding model which has proved to provide a band structure description with submillielectron volt precision throughout the Brillouin zone of binary semiconductors including quantum heterostructures and surface. ${ }^{12,13}$ We model bulk $\mathrm{Al}_{0.3} \mathrm{Ga}_{0.7} \mathrm{As}$ by a linear interpolation of the TB parameters but allow for a parabolic contribution of the relevant twocenter parameters determining the experimental band positions at the $\Gamma, X$, and $\mathrm{L}$ points. Strain effects are taken into account in the same way of smaller TB models using a generalisation of Harrison's $\mathrm{d}^{-2}$ law for hopping integrals known to be reliable for strained III-V QW structures. ${ }^{14}$ The valence band offsets between material constituents are taken from recent $a b$ initio calculations. ${ }^{15}$ The optical dipole matrix elements are derived from the TB Hamiltonian. ${ }^{16} \mathrm{We}$ first tested our TB model by calculating the electronic structure of the InAs ML in bulk GaAs and found a very good agreement with the experimental findings. ${ }^{4-8}$ Fig. 1 shows the simulated optical transitions and PL peak energies at low temperature $(10 \mathrm{~K})$ of a single InAs ML inserted at different positions of a $16 \mathrm{~nm}$-thick GaAs well. The energy subbands 


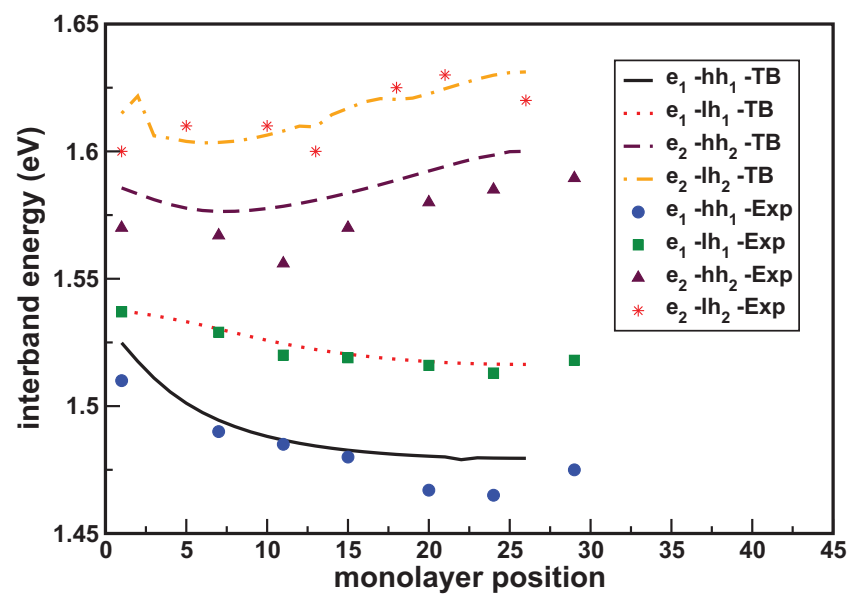

FIG. 1. Calculated and experimental interband energy transitions and InAsrelated photoluminescence in $16 \mathrm{~nm}$-thick GaAs/AlGaAs multiple quantum wells as function of the position of InAs monolayer from the barrier (in MLs).

are calculated at the $\Gamma$-point and labelled according to their dominant bulk-state component: conduction (e), heavy-hole (hh), and light-hole (lh). The agreement is quantitatively good: experimental points lay on the theoretical curve if we assume an error of $10 \%$ on the InAs thickness and position. Implicit in this figure is the assumption that the excitonic binding energy preserves its well properties after the introduction of the monolayer. For the e1-hh1 transition, the red energy shift is maximal when the probe is localised at midwell $(8 \mathrm{~nm})$ and in less extent for e1-lh1. Explanation can be illustrated from the calculation of the charge densities as shown in Fig. 2. The largest amplitude of the ground-state wave functions (Fig 2(a)) is at mid-well and perturbation due to the InAs ML is maximal (Fig. 2(b)). The effect is stronger for hh1 than for $\mathrm{e} 1$ and $\mathrm{lh} 1$, which is a direct consequence of quantum confinement properties correlated to the electron and hole effective masses. Conversely, the first excited state $\mathrm{e} 2$ is strongly affected by the perturbing potential near interfaces (Fig. 2(c)) in the surrounding of the crest of the unperturbed wave functions. The latter result evidences a way to control the intersubband energy process in the conduction band, the probe acting differently for the electron subbands,

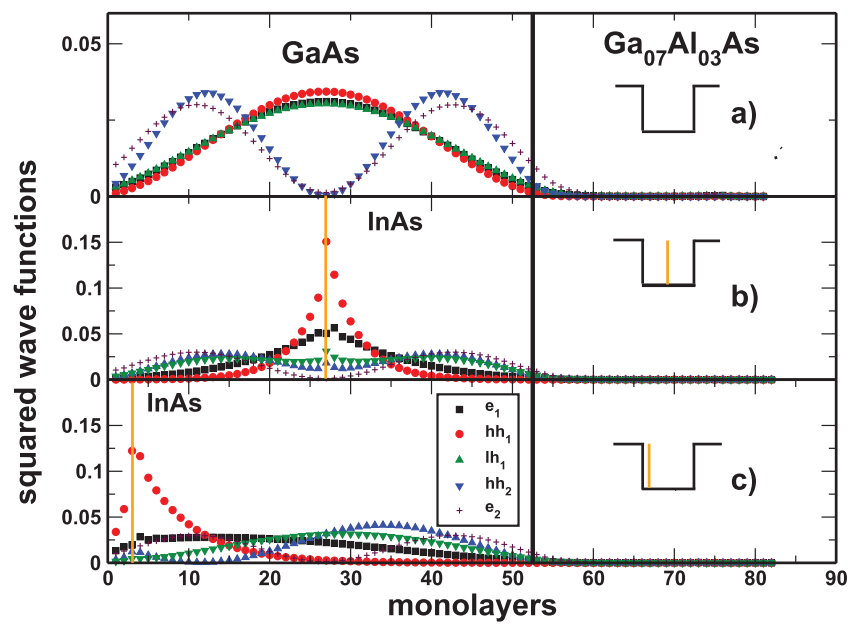

FIG. 2. Calculated hole and electron-state wave functions of a 16-nm thick GaAs/AlGaAs quantum well: without InAs insertion (a), with the InAs ML inserted at mid-well (b) and at $0.6 \mathrm{~nm}$ away from the barrier (c). in agreement with recent observations of the AlAs SML insertions in GaAs quantum wells. We would like to point out the GaAs/AlGaAs QW allows for a better localization of InAs-related states than bulk $\mathrm{GaAs}^{4}$ and excitonic properties of optical spectra should be enhanced.

Symmetry properties of the wave-functions result in a polarization dependence of the optical transitions. The heavy-hole character of the fundamental band edge with the probe leads to optical input that is mainly contributed by the transverse electric (TE) mode whereas the transverse magnetic (TM) mode is depressed. Fig. 3 reports on the dipole matrix elements squared $\left(E_{P}\right.$ in $\left.e V\right)$ between the first $\Gamma$-like valence and conduction band states for TE polarisation of the structures previously studied in Fig. 2. In the k.p approximation, the selection rules, assuming parabolic dispersions of the subbands, allow for the following transitions: ${ }^{17} \mathrm{e} 1-$ hh1, e2-hh2, e1-lh1, e2-lh2, with definite ratio between their optical dipoles. This is different in our tight-binding calculations that show in particular a violation of the $\Delta \mathrm{n}=0$ selection rule for optical transitions. It can be seen in Fig. 3 that the strongly allowed e1-hh1 transitions dominate the low energy range of the absorption spectra with a value of $E_{p}$ nearly constant whatever the position of the probe. In the opposite, the oscillator strengths of the e1-lh1 and parity-forbidden e1-hh2 transitions are very sensitive to the structure of the well. These features can be understood by symmetry considerations. The unperturbed QW (Fig. 3(a)) and with the InAs ML located at mid-well (Fig. 3(b)) are characterized by the D2d point group where the $\mathrm{lh}_{\mathrm{n}}$ and $\mathrm{hh}$ subbands belong to same the representation if $\mathrm{n}+\mathrm{m}$ is odd. ${ }^{18}$ These states are coupled with strength inversely proportional to their energy separation, as defined by perturbation theory, and the resulting optical matrix elements evolve from TE contributions both of the heavy-holes and lightholes zinc-blende periodic functions. For instance, the e1-hh2 transition displays a light-hole character in our calculations, which is also evidenced from the wave functions of hh2 and lh1 in Figs. 2(b) and 2(c). These considerations explain the appearance of parity-forbidden transitions in Fig. 3(b) which are too weak in Fig. 3(a) to be shown up. For

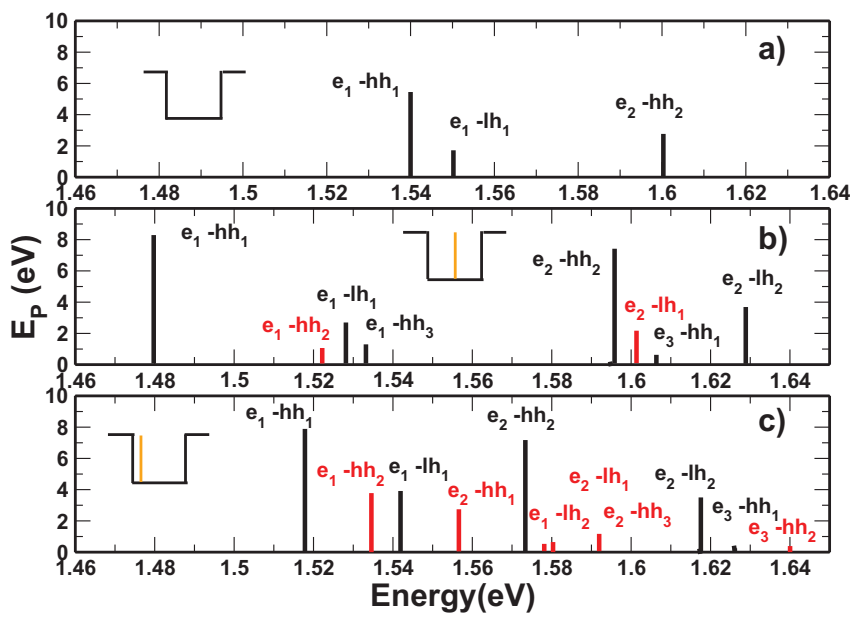

FIG. 3. Calculated dipole matrix element squared (Ep in eV) for transverse electric polarization between the $\Gamma$-like valence and conduction subbands of a 16-nm thick GaAs quantum well: without InAs insertion (a), with the InAs ML inserted at mid-well (b), and at $0.6 \mathrm{~nm}$ away from the barrier (c). 


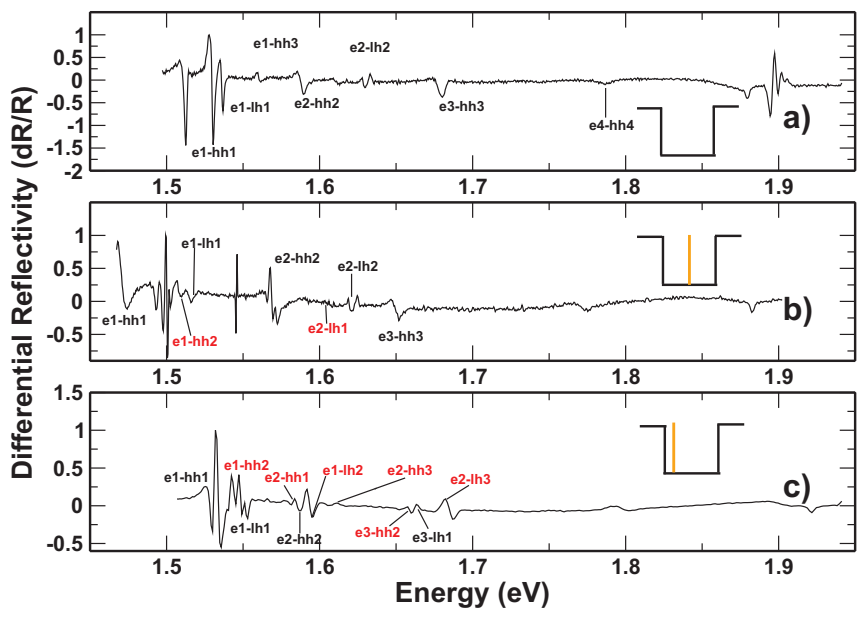

FIG. 4. Differential reflectivity measurements of 16-nm thick GaAs quantum wells: without InAs insertion (a), with the InAs ML inserted at mid-well (b), and at $0.6 \mathrm{~nm}$ away from the barrier (c).

the probe located near the QW interface (Fig. 3(c)), the structure is asymmetric with a lower point symmetry group $\mathrm{C}_{2 \mathrm{v}}$ where all states belong to the same irreducible representation $^{18}$ and are coupled to each other. Consequently, the parityforbidden transitions are enforced compared to Fig. 3(b).

To validate these simulations, we have performed photo-reflectance (PR) spectroscopy which is known to be a valid approach to investigate the optical properties of QWs in a large energy range. The growth of the multi quantumwell structures was performed by conventional solid-source molecular beam epitaxy. ${ }^{9}$ Samples consist of six GaAs wells of $16 \mathrm{~nm}$ thick separated by $8.5 \mathrm{~nm}$ of $\mathrm{Al}_{0.3} \mathrm{Ga}_{0.7} \mathrm{As}$ barriers where a single InAs monolayer was inserted at different positions in the well. Reflectivity measurements were made using experimental setup where the focused image of a $250 \mathrm{~W}$ tungsten lamp was reflected onto the input slit of a $28 \mathrm{~cm}$ focal-length monochromator, and dispersed and detected with a silicium photodiode followed by a lock-in amplifier. The optical transition energy at low temperature $(10 \mathrm{~K})$ was determined from the reflectivity measurements. Figure 4 shows the differential reflectivity data obtained for the reference sample (without monolayer) and the samples with one InAs monolayer inserted in the wells at $8 \mathrm{~nm}$ and $0.6 \mathrm{~nm}$ away from the barrier. Strong and extended signals are observed with the probe and confirm the optical spectra of Fig. 3. In particular, the stronger featured structure is clearly due to the e1-hh1 excitonic transition and the other peaks can be precisely assigned by comparison with the present modelling.

Finally, we calculated the in-plane effective masses for the hh1 states of the perturbed structures and found a low value of about $0.18 \mathrm{~m} 0$ ( $\mathrm{m} 0$ is the free electron mass) in a wide energy range independently of the position of the probe. The improved confinement of carriers and optical excitations together with low effective mass band in ultrathin InAs quantum-wells grown on $\operatorname{GaAs}(001)$ can be efficiently used in separate confinement heterostructure ( $\mathrm{SCH}$ ) laser diodes at different wavelengths to lower the threshold current density of laser generation and increase the power of $\mathrm{SCH}$ lasers operating at room temperature. For the probe located near the QW interface, the absorption spectrum is expanded around the wavelength of $808 \mathrm{~nm}$ which is important for applications in solid state lasers.

In conclusion, we have studied the optical properties of ultrathin InAs layers in GaAs quantum wells and shown that a strong and stable optical process can occur at wavelengths well below $850 \mathrm{~nm}$. Further engineering of the electronic structure could be achieved by considering different well thicknesses and $\mathrm{Al}$ contents in the barrier. Our results show the usefulness of the present system to implement $\mathrm{SCH}$ lasers based on engineered monolayer insertions.

The authors would like to gratefully acknowledge P. Voisin for stimulating discussions.

${ }^{1}$ M. Geiger, A. Bauknecht, F. Adler, H. Schweizer, and F. Scholz, J. Cryst. Growth 170, 558 (1997).

${ }^{2}$ N. N. Ledentsov, M. Grundmann, N. Kirstaedter, O. Schmidt, R. Heitz, J. Böhrer, D. Bimberg, V. M. Ustinov, V. A. Shchukin, P. S. Kop’ev, Zh. I. Alferov, S. S. Ruvimov, A. O. Kosogov, P. Werner, U. Richter, U. Goesele, and J. Heydenreich, Solid-State Electron. 40, 785 (1996).

${ }^{3}$ E. P. O'Reilly and A. Ghiti, in Quantum Well Lasers, edited by P. S. Zory, Jr. (Academic, San Diego, 1993).

${ }^{4}$ O. Brandt, L. Tapfer, R. Cingolani, K. Ploog, M. Hohenstein and F. Phillipp, Phys. Rev. B 41, 12599 (1990).

${ }^{5}$ K. Kim, P. R. C. Kent, A. Zunger, and C. B. Geller, Phys. Rev. B 66, 45208 (2002).

${ }^{6}$ R. C. Iotti, L. C. Andreani, and M. Di Venta, Phys. Rev. B 57, R15072 (1998).

${ }^{7}$ Z. Sun, Z. Y. Xu, Y. Ji, B. Q. Sun, B. R. Wang, S. S. Huang, and H. Q. Ni, Appl. Phys. Lett. 90, 71907 (2007).

${ }^{8}$ A. R. Goñi, M. Stroh, C. Thomsen, F. Heinrichsdorff, V. Türck, A. Krost, and D. Bimberg, Appl. Phys. Lett. 72, 1433 (1998)

${ }^{9}$ J.-Y. Marzin and J.-M. Gerard, Phys. Rev. Lett. 62, 2172 (1989).

${ }^{10}$ C. Mejri, M. Chaouache, M. Maaref, P. Voisin, and J.-M. Gerard, Semicond. Sci. Technol. 21 1018, (2006).

${ }^{11}$ T. Unuma, S. Takata, Y. Sakasegawa, K. Hirakawa, and A. Nakamura, J. Appl. Phys. 109, 043506 (2011).

${ }^{12}$ J.-M. Jancu, J.-C. Girard, M. O. Nestoklon, A. Lemaître, F. Glas, Z. Z. Wang, and P. Voisin, Phys. Rev. Lett. 101, 196801 (2008).

${ }^{13}$ J.-M. Jancu, R. Scholz, G. C. La Rocca, E. A. de Andrada e Silva, and P. Voisin, Phys. Rev. B 70, 121306 (2004).

${ }^{14}$ P. Boring, J.-M. Jancu, B. Gil, D. Bertho, C. Jouanin, and K. J. Moore, Phys. Rev. B 46, 4764 (1992).

${ }^{15}$ Y.-H. Li, A. Walsh, S. Chen, W.-J. Yin, J.-H. Yang, J. Li, J. L. F. Da Silva, X. G. Gong, and S.-H. Wei, Appl. Phys. Lett. 94, 212109 (2009).

${ }^{16}$ T. B. Boykin and P. Vogl, Phys. Rev. B 65, 035202 (2001).

${ }^{17}$ G. Bastard, Wave Mechanics Applied to Semiconductor Heterostructures (Monograph de Physique, Paris, 1988).

${ }^{18}$ D. L. Smith and C. Mailhot, Rev. Mod. Phys. 62, 173 (1990). 